\title{
Characterization of the rice NLA family reveals a key role for OsNLA1 in phosphate homeostasis
}

\author{
Jian Yang ${ }^{1}$, Lan Wang ${ }^{1,2}$, Chuanzao Mao ${ }^{3}$ and Honghui Lin ${ }^{*}$
}

\begin{abstract}
Background: Phosphate (Pi), an essential mineral nutrient for plant development and reproduction, is one of the main components of fertilizers in modern agriculture. Previous research demonstrated that AtNLA1 mediates ubiquitination of Pi transporters in the plasma membrane and triggers their endocytosis and degradation in Arabidopsis. In this study, we researched the function of NLA homologous proteins in Pi homeostasis in rice.

Findings: Two OsNLA homologs from rice (Oryza sativa L.) were identified by bioinformatics and phylogenetic analysis and designated OsNLA1 and OsNLA2. The OsNLA1 clustered with Arabidopsis AtNLA1, was expressed higher than OsNLA2 and was transcriptionally repressed under Pi-deficient condition. Loss-of-function of OsNLA1 caused $P$ overaccumulation and growth inhibitions in both root and shoot under Pi-sufficient condition. Furthermore, mutation of OSNLA1 affected expression of Pi tranporters and root hair development under Pi-sufficient and/or Pi-deficient conditions.
\end{abstract}

Conclusions: OsNLA1 plays a key role in maintaining phosphate homeostasis in rice.

Keywords: Rice, Phosphate, OsNLA1, Pi-homeostasis

\section{Findings}

Phosphorus (P) is a mineral nutrient essential for plant development and reproduction, and is integral to several macromolecules such as phospholipids and nucleic acids. Despite the indispensable role of $P$ for plants, levels of phosphate (orthophosphate; $\mathrm{Pi}$ ), the only form of $\mathrm{P}$ that can be taken up by plants, are commonly limited because of chemical fixation and microbial activity (Raghothama, 1999). To cope with suboptimal Pi conditions, plants have developed a series of adaptive responses, such as induction of Pi transporters and modification of root system architecture (Raghothama, 1999; Lin et al., 2009; Wu et al., 2013). Plant uptake of Pi is largely mediated by plasma membrane -localized Pi transporters belonging to the PHOSPHATE TRANSPORTER1 (PT) symporter family. Thirteen $P T$ genes have been identified in rice (Oryza sativa) and nine in Arabidopsis thaliana (Goff et al., 2002; Karthikeyan et al., 2002). OsPTs differ in tissue expression

\footnotetext{
* Correspondence: hhlin@scu.edu.cn

${ }^{1}$ Ministry of Education Key Laboratory for Bio-Resource and Eco-Environment, College of Life Science, Sichuan University, Chengdu 610064, China Full list of author information is available at the end of the article
}

patterns and affinities for Pi, resulting in diverse functions in plants. For instance, the high-affinity $\mathrm{Pi}$ transporter OsPT8 is universally expressed in rice, and is responsible for half of its Pi uptake (Chen et al., 2011; Jia et al., 2011). Although most OsPTs in rice are induced at the transcriptional level by Pi starvation or mycorrhizal symbiosis (Yang et al., 2012; Secco et al., 2013), post-transcriptional regulating of OsPT family proteins is also important to their activities (Gonzalez et al., 2005; Bayle et al., 2011; Chen et al., 2011; Chen et al., 2015). NITROGEN LIMITATION ADAPTATION (NLA), designated AtNLA1 in this study, was first identified as a positive regulator for the adaptability of Arabidopsis to nitrogen limitation (Peng et al., 2007), and later analysis of Pi concentration revealed that the early senescence phenotype of atnla mutant plants was due to Pi toxicity (Kant et al., 2011). In Arabidopsis, AtNLA1 can interact with AtPTs members via its SPX domain, and mediate ubiquitination of AtPTs in the plasma membrane and trigger their endocytosis and degradation (Lin et al., 2013; Park et al., 2014). Recently, two research groups separately reported roles of OsNLA1 in mantaining Pi homeostasis in rice (Yue et al., 
2017; Zhong et al., 2017). Yue et al., (2017) additionally reported OsNLA1 functioned as a ubiquitin ligase to degrade Pi transporters in rice, with a similar function of AtNLA1 in Arabidopsis.. In this study, we were interested in the phylogenetic relationship of the NLA family and expression of OsPTs and root hair development in osnla1 mutant.

An unrooted phylogenetic analysis of the NLA family proteins with four monocots ( $B$. distachyon, $S$. bicolor, S. italica and rice) and five dicots (grapevine, soybean, apple, M. truncatula and Arabidopsis), revealed the presence of two distinct clades. Although all plants had proteins belonging to clade I, in which AtNLA1 involved in regulating $\mathrm{Pi}$ homeostasis was present (Kant et al., 2011), all monocots and only some dicots had NLA members belonging to clade II (Fig. 1a). This suggested that NLA members of clade I conservatively functioned in maintaining Pi homeostasis among different plant species. Quantitative reverse-transcription PCR (qRT-PCR) was performed on different tissues for rice plants grown in nutrient solutions under Pi- sufficient $(300 \mu M)$ condition (Additional file 1). The amplification efficiencies of gene-specific primers for OsNLA1 and OsNLA2 were assessed and found that they were approximately equal $(95.5 \%$ for OsNLA1 and $94.3 \%$ for OsNLA2) (Additional file 2: Figure S1 and Additional file 3: Table S1). Then, the transcript level of OsNLA1 and OsNLA2 in plants were compared, and found that the transcript level of OsNLA1 was higher than that of OsNLA2 in all tissues tested, with about 1.5-fold in shoot base, 4.5-fold in root and 80-fold in leaf sheath (Fig. 1b and c). Previous transcriptome analysis also shown that OsNLA1 abounace was higher than OsNLA2 in both root and shoot (Secco et al., 2013). Furthermore, the OsNLA1 transcript was differentially regulated by $\mathrm{Pi}$ availability, with higher expression in $\mathrm{Pi}$ - sufficient and lower expression in $\mathrm{Pi}$-deficient conditions (Fig. 1d), as AtNLA1 in Arabidopsis (Lin et al., 2013); however, the OsNLA2 transcripts remained at relatively constant levels (Fig. 1e). The transcriptional change of OsNLA1 in response to $\mathrm{Pi}$ supply was also identified by RNA sequencing (Secco et al., 2013). Based on phylogenetic relationships and expression levels and responses to Pi starvation of the $N L A$ family in rice, we suggest that OsNLA1 might plays a major role in regulating $\mathrm{Pi}$ homeostasis, as does AtNLA1 in Arabidopsis.

To characterize the functions of the NLA gene family in rice, we searched for publicly available mutants in different rice genomic resources. One T-DNA null mutant in OsNLA1 gene (PFG_1B-12,301) was obtained from RISD DB (Rice T-DNA Insertion Sequence Database) (Fig. 2a and b). After growth for 30 d under Pi-sufficient condition $(300 \mu \mathrm{M} \mathrm{Pi} ;+\mathrm{P})$, the shoots and roots of osnlal were inhibited compared with wild-type (WT) plants (Fig. 2c and d). In addition, osnla1 displayed leaf tip necrosis on old leaves, which was a typical $\mathrm{Pi}$ toxicity symptom in rice (Fig. 2c, Additional file 2: Figure S1). This symptom in osnla1 was not observed when grown in Pideficient condition $(10 \mu \mathrm{M} \mathrm{Pi}$; $-\mathrm{P})$. Moreover, the inhibited root phenotype of Osnla1 was reversed when grown in Pi-deficient condition (Fig. 2c and d). Total $\mathrm{P}$ concentrations in all tissues of osnla1 were higher than of WT, with 1.24-fold in roots and 1.46fold in leaves under Pi-sufficient condition (Fig. 2e). This indicating that OsNLA1 played a key role in $\mathrm{Pi}$ uptake in rice, as previously reported (Yue et al., 2017; Zhong et al., 2017). However, under Pi-deficient condition, total P concentrations in old leaves (leaves 2 and 3) of osnla1 were decreased by $17-25 \%$, while total $\mathrm{P}$ concentrations in youngest leaves (leaves 7 ) of osnla1 were increased by $21 \%$ compared with WT. The total $\mathrm{P}$ distribution rate in WT plants grown in Pi-deficient condition was 1.68 -fold higher than that in plants grown in Pi-sufficient condition. However, the rate in osnla1 mutants grown in Pi-deficient condition was 3.19-fold higher than that in plants grown in Pi-sufficient condition (Fig. 2f). This significant increased total $\mathrm{P}$ distribution rate under $\mathrm{Pi}$ limiting condition sustained the newly leaves growth (Fig. 2c and d). $\mathrm{Pi}$ is the major form of $\mathrm{P}$ transported within the plants, and old leaves $\mathrm{Pi}$ pool would be the source of $\mathrm{Pi}$ in regard to young leaves under Pi-deficient condition, resulting in higher $\mathrm{P}$ concentration in young leaves ( $\mathrm{Li}$ et al., 2015, b). Thus, these results indicated that OsNLA1 was also involved in $\mathrm{Pi}$ remobilization besides $\mathrm{Pi}$ uptake. This was expected because OsPT1 and OsPT8 also function in the redistribution of Pi from source to sink organs (Sun et al., 2012; Li et al., 2015, b). In a recent study, AtNLA1 was also involved in mediating degradation of NRT1.7 and further remobilizing nitrate from source to sink in Arabidopsis (Liu et al., 2016). Whether OsNLA1 also functions in nitrate remobilization need further studies.

As plants may modify root system architecture when growth in suboptimal Pi condition, we analyzed root hairs when osnla1 and WT were grown in Pi-sufficient and -deficient conditions. After Pi-deficient growth for $10 \mathrm{~d}$, WT developed many root hairs (Fig. 3a and b), as previously reported (Zhou et al., 2008; Sun et al., 2012). However, the length of root hairs on osnla1 mutant was 1.5-fold those of WT plants grown in Pi-deficient condition. Furthermore, osnla1 mutant also had increased root hairs length under Pi-sufficient condition compared with WT plants. Since OsNLA1 could mediate 


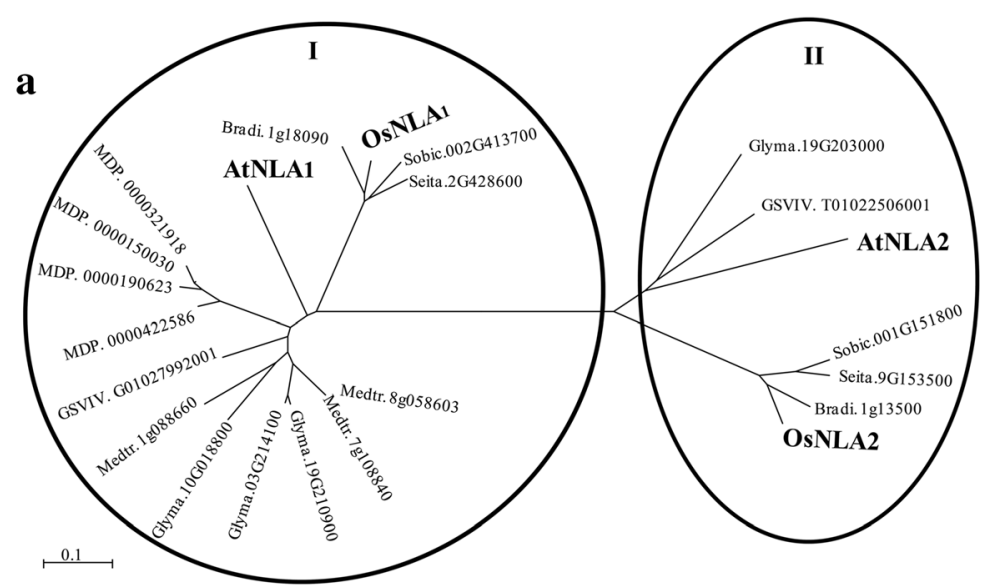

b
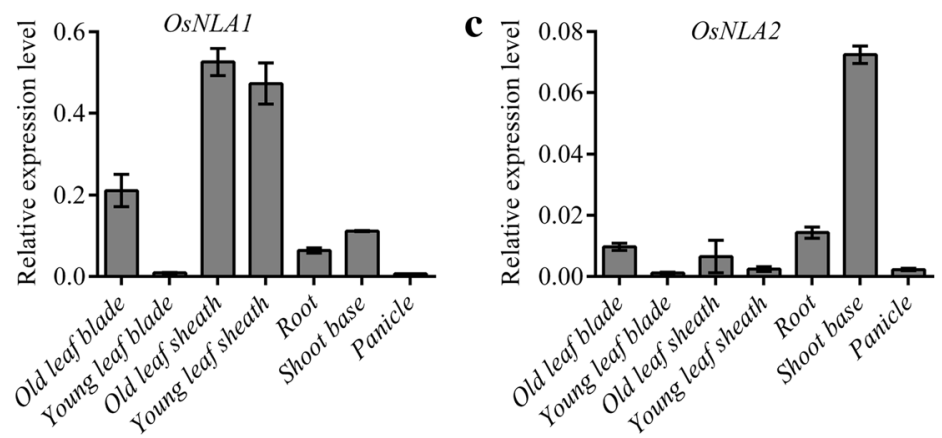

d

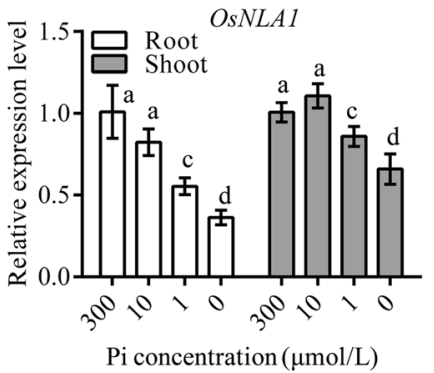

e

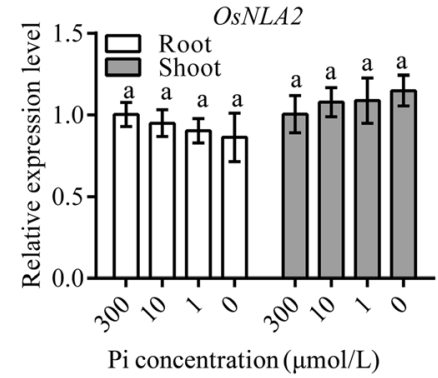

Fig. 1 Phylogenetic relationships of NLA proteins and OsNLA family expression patterns in rice. a Unrooted phylogenetic tree of the NLA family using MEGA 5.10 by the neighbor-joining method. Dicots: Vitis vinifera (GSVIV), Glycine max (Glyma), Malus domestica (MDP), Medicago truncatula (Medtr), Arabidopsis thaliana (At); monocots: Brachypodium distachyon (Bradi), Sorghum bicolor (Sobic) and Setaria italica (Si). OsNLA family expression patterns in rice. b, c Spatial expression of OsNLAs transcripts. Transcript levels for OsNLA1 (b) and OSNLA2 (c)in leaf sheath, leaf blade, roots and shoot base in 30-d-old seedlings grown in nutrient solutions containing $300 \mu \mathrm{M} \mathrm{Pi}$ and in panicle before flowering. Expression of OsNLA1 and OSNLA2 is relative to OSACTIN2. d, e Transcript levels of OSNLA1 (d) and OSNLA2 (e) in root and shoot of plants grown for $10 \mathrm{~d}$ in nutrient solutions with different $\mathrm{Pi}$ concentrations. Data represent mean \pm SD of three replicates. Different letters represent significant differences according to Duncan's multiple range test $(P<0.05)$

the degradation of OsPT2 and OsPT8 (Yue et al., 2017), inhibition of root growth and induce of root hair in osnla1 was expected because OsPTs were involved in regulating root growth and root hair development (Jia et al., 2011; Sun et al., 2012).

Since changing the expression of OsPT4 or OsPT8 affects the expression of other Pi transporters in rice (Jia et al., 2011; Sun et al., 2012; Li et al., 2015, b) and protein levels of OsPT2 and OsPT8 accumulated in osnla1 mutants (Yue et al., 2017), we then analyzed the transcriptional levels of OsPTs following WT and osnla1 mutant growth in Pi-sufficient and -deficient conditions for 10d. In the shoot of osnla1 mutant, transcripts of most of Pi transporters were induced (Fig. 3c). Compared with the WT, expressions of OsPT6 and OsPT8 were greatly induced under both Pi-sufficient and -deficient conditions. Although OsPT2, OsPT4 and OsPT10 were also upregulated under $\mathrm{Pi}$-sufficient condition, their transcript levels did not change under Pi-deficient condition. Expression of OsPT1 was induced only when osnla1 mutant was 

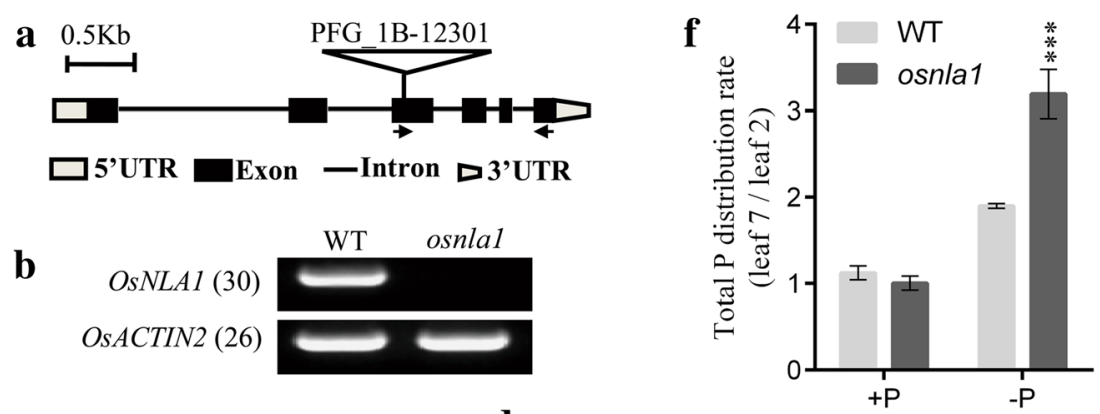

c
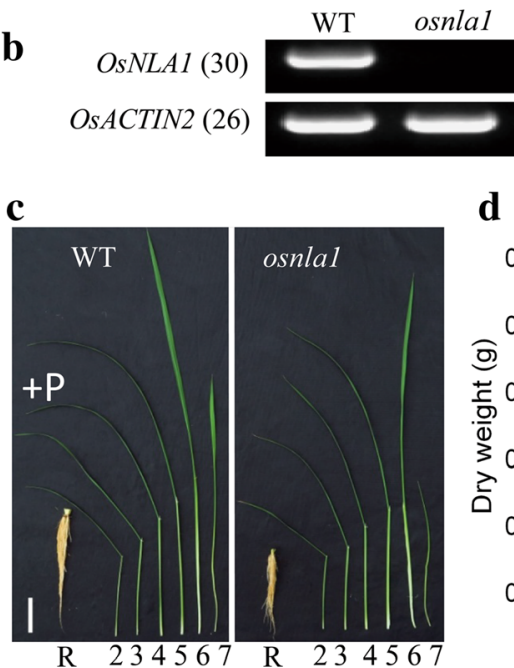

d

$\mathbf{e}$
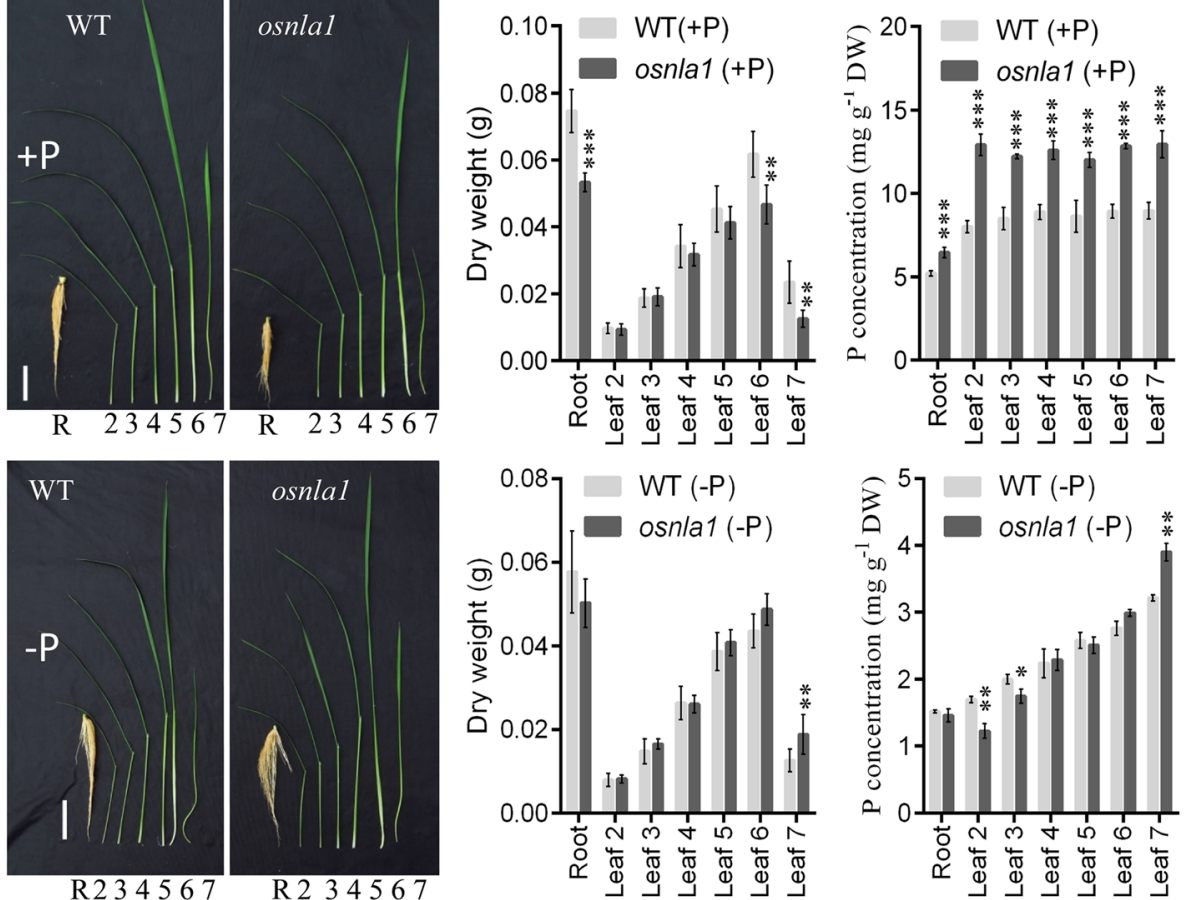

Fig. 2 Phenotypical characteristics of osnla1 mutant. a Genomic structure of rice OsNLA1. Position of the T-DNA insertion in OsNLA1 is indicated by a triangle. Small arrows are the gene-specific primers for RT-PCR. $\mathbf{b}$ RT-PCR analysis of OsNLA1 expression in roots of the mutant and wild-type (Dongjin; WT). c Phenotype comparison between WT and osnla1 mutant. 30-d-old WT and osnla1 grown under Pi-sufficient (300 $\mu \mathrm{M} ;+$ P; upper) and Pi-deficient (10 $\mu \mathrm{M}$; -P; lower) conditions. Bars $=5 \mathrm{~cm}$. d Biomass of 30-d-old WT and osnla1 in (c). Data represent mean \pm SD of eight replicates. e Total P concentration in different leaves and roots of 30-d-old WT and osnla1 in (c). Data represent mean \pm SD of three replicates. $\mathbf{f}$ Distribution ratio of total $P$ between young leaves (leaf 7 ) and old leaves (leaf 2) in osnla1 and WT. Asterisks represent a significant difference with the corresponding WT $\left(^{* *}, P<0.01 ; * *, P<0.001\right)$

grown under Pi-deficient condition. However, contrary to our finding, Yue et al. (2017) found that OsPT2 and OsPT8 were unchanged in leaf under Pi-sufficient conditions. This might be resulted from transcriptional levels of OsNLA1 and Pi transporters differed in various tissues (Fig. 1b; Remy et al., 2012). Unlike $\mathrm{Pi}$ transporters induced in the shoot, transcripts of $\mathrm{Pi}$ transporters were differentially regulated under Pi-sufficient and -deficient conditions in root of osnla1 mutant (Fig. 3c). The transcriptional levels of OsPT1 and OsPT4 were induced under Pi-sufficient condition, but unchanged under Pi-deficient condition. In contrast, OsPT6, OsPT8 and OsPT10 were downregulated under Pi-deficient condition, but unchanged under Pi-sufficient condition. The increased or repressed expression of these $\mathrm{Pi}$ transporters was caused, at least in part, by accumulated protein level of Pi transporters in osnla1 mutant, because changing the expression of OsPT4 or OsPT8 affects the expression of Pi transporters in rice (Jia et al., 2011; Zhang et al., 2015). Moreover, induced expression of OsPT1 and OsPT8 in shoot of osnla1 mutant under Pi-deficient condition would further remobilize Pi from old to young leaves (Sun et al., 2012; Li et al., 2015, b). 

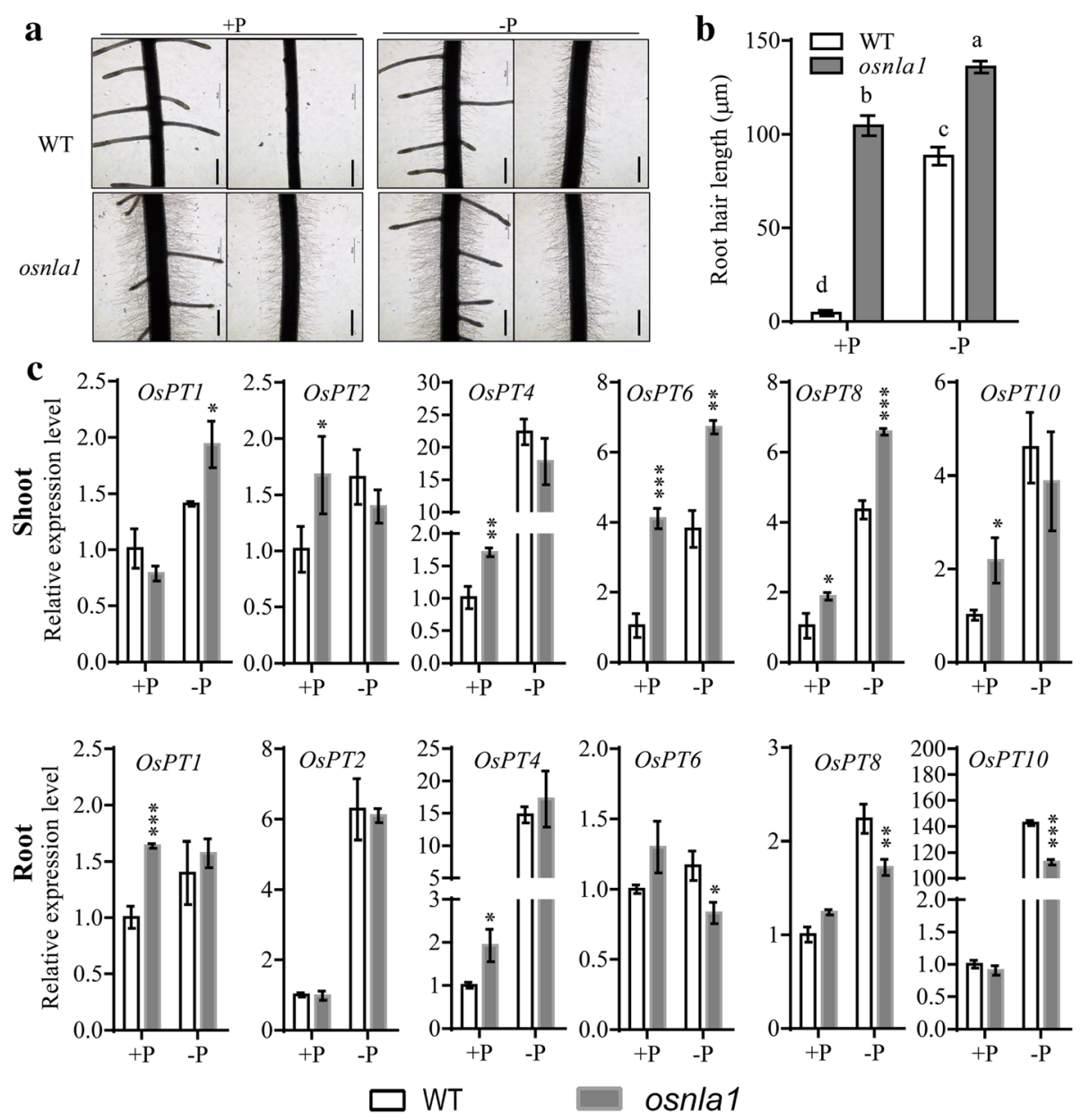

Fig. 3 Root hair proliferation and expression of Pi transporter genes in osnla1 mutant and wild-type (WT). a Root hair proliferation of WT and osnla1 grown on Pi-sufficient (+P; left) and Pi-deficient (-P; right). Bars $=100 \mu \mathrm{m}$. b Root hair length in the maturation zone of roots. Data represent mean \pm SD of eight replicates. Different letters represent significant differences according to Duncan's multiple range test $(P$ < 0.05). c Expression of Pi transporter genes in osnla1 mutant and WT. Ten-day-old plants grown in Pi-sufficient (300 $\mu \mathrm{M}$ Pi) nutrient solution were transferred to Pi-sufficient $(+P)$ and -deficient $(-P)$ conditions for $10 \mathrm{~d}$. RNA was extracted from shoots (upper) and roots (lower) for qRT-PCR. Data represent mean \pm SD of three replicates. Asterisks represent significant difference with the corresponding WT $\left({ }^{*}, P<0.05 ;{ }^{* *}, P<0.01 ; * * *, P<0.001\right)$

Since OsNLA1 mediates degradation of OsPTs and plays a key role in maintaining Pi homeostasis in rice. In this research, we identified OsNLA1 could regulate root system architecture, Pi transporters at the transcriptional levels and Pi redistribution from source to sink organs. These results presented here will provide a novel insight into the function of OsNLA1 in rice.

\section{Additional files}

Additional file 1: Materials and methods. (DOCX $19 \mathrm{~kb}$ )

Additional file 2: Figure S1. Calculation of PCR efficiencies. Figure S2. Leaf blades of 30-d-old WT and osnla1 grown under Pi-sufficient (300 $\mu \mathrm{M}$; $+\mathrm{P})$ and Pi-deficient (10 $\mu \mathrm{M} ;-\mathrm{P})$ conditions. (PPTX $304 \mathrm{~kb})$

Additional file 3: Table S1. Primers used in this study. (DOCX $14 \mathrm{~kb}$ )

\section{Abbreviations}

NLA: Nitrogen Limitation Adaptation; Pi: Phosphate: PT: Phosphate transporter; RISD DB: Rice T-DNA Insertion Sequence Database; WT: Wild type

\section{Acknowledgements}

This work was supported by the National Natural Science Foundation of China (31701984), Foundation of Sichuan University (2017SCU12007) and National Key Research and Development Program of China (2016YFD0100700)

\section{Authors' Contributions}

$\mathrm{HHL}, \mathrm{CZM}$ and JY designed the experiments. JY developed relevant research materials and performed the experiments together with LW. HHL, CZM and $J Y$ wrote the manuscript. All authors read and approved the final manuscript.

\section{Competing Interests}

The authors declare that they have no competing interests. 


\section{Publisher's Note}

Springer Nature remains neutral with regard to jurisdictional claims in published maps and institutional affiliations.

\section{Author details}

${ }^{1}$ Ministry of Education Key Laboratory for Bio-Resource and Eco-Environment, College of Life Science, Sichuan University, Chengdu 610064, China. ${ }^{2}$ Biogas Institute of Ministry of Agriculture, Chengdu 610041, China. ${ }^{3}$ State Key Laboratory of Plant Physiology and Biochemistry, College of Life Sciences, Zhejiang University, Hangzhou 310058, China.

Received: 15 July 2017 Accepted: 12 December 2017

Published online: 28 December 2017

\section{References}

Bayle V, Arrighi JF, Creff A, Nespoulous C, Vialaret J, Rossignol M, Gonzalez E, Paz-Ares J, Nussaume L (2011) Arabidopsis thaliana high-affinity phosphate transporters exhibit multiple levels of posttranslational regulation. Plant Cell 23:1523-1535

Chen J, Liu Y, Ni J, Wang Y, Bai Y, Shi J, Gan J, Wu Z, Wu P (2011) OsPHF1 regulates the plasma membrane localization of low- and high-affinity inorganic phosphate transporters and determines inorganic phosphate uptake and translocation in rice. Plant Physiol 157:269-278

Chen J, Wang Y, Wang F, Yang J, Gao M, Li C, Liu Y, Yamaji N, Ma JF, Paz-Ares J, Nussaume L, Zhang S, Yi K, Wu Z, Wu P (2015) The rice CK2 kinase regulates trafficking of phosphate transporters in response to phosphate levels. Plant Cell 27:711-723

Goff SA, Ricke D, Lan T-H, Presting G, Wang R, Dunn M, Glazebrook J, Sessions A, Oeller P, Varma H, Hadley D, Hutchison D, Martin C, Katagiri F, Lange BM, Moughamer T, Xia Y, Budworth P, Zhong J, Miguel T, Paszkowski U, Zhang S, Colbert M, W-I S, Chen L, Cooper B, Park S, Wood TC, Mao L, Quail P, Wing R, Dean R, Yu Y, Zharkikh A, Shen R, Sahasrabudhe S, Thomas A, Cannings R, Gutin A, Pruss D, Reid J, Tavtigian S, Mitchell J, Eldredge G, Scholl T, Miller RM, Bhatnagar S, Adey N, Rubano T, Tusneem N, Robinson R, Feldhaus J, Macalma T, Oliphant A, Briggs S (2002) A draft sequence of the rice genome (Oryza sativa L. ssp. japonica). Science 296:92-100

Gonzalez E, Solano R, Rubio V, Leyva A, Paz-Ares J (2005) PHOSPHATE TRANSPORTER TRAFFIC FACILITATOR1 is a plant-specific SEC12-related protein that enables the endoplasmic reticulum exit of a high-affinity phosphate transporter in Arabidopsis. Plant Cell 17:3500-3512

Jia H, Ren H, Gu M, Zhao J, Sun S, Zhang X, Chen J, Wu P, Xu G (2011) The phosphate transporter gene OsPht1;8 is involved in phosphate homeostasis in rice. Plant Physiol 156:1164-1175

Kant S, Peng M, Rothstein SJ (2011) Genetic regulation by NLA and microRNA827 for maintaining nitrate-dependent phosphate homeostasis in Arabidopsis. PLoS Genet 7:e1002021

Karthikeyan AS, Varadarajan DK, Mukatira UT, D'Urzo MP, Damsz B, Raghothama KG (2002) Regulated expression of Arabidopsis phosphate transporters. Plant Physiol 130:221-233

Li XM, Chao DY, Wu Y, Huang X, Chen K, Cui LG, Su L, Ye WW, Chen H, Chen HC, Dong NQ, Guo T, Shi M, Feng Q, Zhang P, Han B, Shan JX, Gao JP, Lin HX (2015) Natural alleles of a proteasome alpha2 subunit gene contribute to thermotolerance and adaptation of African rice. Nat Genet 47:827-833

Li Y, Zhang J, Zhang X, Fan H, Gu M, Ou H, Xu G (2015) Phosphate transporter OsPht 1;8 in rice plays an important role in phosphorus redistribution from source to sink organs and allocation between embryo and endosperm of seeds. Plant Sci 230:23-32

Lin WY, Huang TK, Chiou TJ (2013) NITROGEN LIMITATION ADAPTATION, a target of MicroRNA827, mediates degradation of plasma membrane-localized phosphate transporters to maintain phosphate homeostasis in Arabidopsis. Plant Cell 25:4061-4074

Lin WY, Lin SI, Chiou TJ (2009) Molecular regulators of phosphate homeostasis in plants. J Exp Bot 60:1427-1438

Liu W, Sun Q, Wang K, Du Q, Li WX (2016) Nitrogen Limitation Adaptation (NLA) is involved in source-to-sink remobilization of nitrate by mediating the degradation of NRT1.7 in Arabidopsis. New Phytol 214:734-744

Park BS, Seo JS, Chua NH (2014) NITROGEN LIMITATION ADAPTATION recruits PHOSPHATE2 to target the phosphate transporter PT2 for degradation during the regulation of Arabidopsis phosphate homeostasis. Plant Cell 26:454-464
Peng M, Hannam C, Gu H, Bi YM, Rothstein SJ (2007) A mutation in NLA, which encodes a RING-type ubiquitin ligase, disrupts the adaptability of Arabidopsis to nitrogen limitation. Plant J 50:320-337

Raghothama KG (1999) Phosphate acquisition. Annu Rev Plant Biol 50:665-693

Remy E, Cabrito TR, Batista RA, Teixeira MC, Sa-Correia I, Duque P (2012) The Pht1;9 and Pht1;8 transporters mediate inorganic phosphate acquisition by the Arabidopsis thaliana root during phosphorus starvation. New Phytol 195: 356-371

Secco D, Jabnoune M, Walker H, Shou H, Wu P, Poirier Y, Whelan J (2013) Spatiotemporal transcript profiling of rice roots and shoots in response to phosphate starvation and recovery. Plant Cell 25:4285-4304

Sun S, Gu M, Cao Y, Huang X, Zhang X, Ai P, Zhao J, Fan X, Xu G (2012) A constitutive expressed phosphate transporter, OsPht1;1, modulates phosphate uptake and translocation in phosphate-replete rice. Plant Physiol 159:1571-1581

Wu P, Shou H, Xu G, Lian X (2013) Improvement of phosphorus efficiency in rice on the basis of understanding phosphate signaling and homeostasis. Curr Opin Plant Biol 16:205-212

Yang SY, Gronlund M, Jakobsen I, Grotemeyer MS, Rentsch D, Miyao A, Hirochika H, Kumar CS, Sundaresan V, Salamin N, Catausan S, Mattes N, Heuer S, Paszkowski U (2012) Nonredundant regulation of rice arbuscular mycorrhizal symbiosis by two members of the phosphate transporter 1 gene family. Plant Cell 24:4236-4251

Yue W, Ying Y, Wang C, Zhao Y, Dong C, Whelan J, Shou H (2017) OsNLA1, a RING-type ubiquitin ligase, maintains phosphate homeostasis in Oryza sativa via degradation of phosphate transporters. Plant J 90:1040-1051

Zhang F, Sun Y, Pei W, Jain A, Sun R, Cao Y, Wu X, Jiang T, Zhang L, Fan X, Chen A, Xu G, Sun S (2015) Involvement of OsPht1;4 in phosphate acquisition and mobilization facilitates embryo development in rice. Plant J 82:556-569

Zhong S, Mahmood K, Bi YM, Rothstein SJ, Ranathunge K (2017) Altered expression of OsNLA1 modulates Pi accumulation in rice (Oryza sativa L.) plants. Front Plant Sci 8: 928

Zhou J, Jiao F, Wu Z, Li Y, Wang X, He X, Zhong W, Wu P (2008) OsPHR2 is involved in phosphate-starvation signaling and excessive phosphate accumulation in shoots of plants. Plant Physiol 146:1673-1686

\section{Submit your manuscript to a SpringerOpen ${ }^{\circ}$ journal and benefit from:}

- Convenient online submission

- Rigorous peer review

- Open access: articles freely available online

- High visibility within the field

Retaining the copyright to your article

Submit your next manuscript at $>$ springeropen.com 